\title{
Supplementation of pancreatic digestive enzymes alters the composition of intestinal microbiota in mice
}

\section{$\operatorname{AUTHOR}(\mathrm{S})$ :}

Nishiyama, Hiroki; Nagai, Tomoyuki; Kudo, Masatoshi; Okazaki, Yoshihisa; Azuma, Yoshinao; Watanabe, Tomohiro; Goto, Susumu; Ogata, Hiroyuki; Sakurai, Toshiharu

\section{CITATION:}

Nishiyama, Hiroki ...[et al]. Supplementation of pancreatic digestive enzymes alters the composition of intestinal microbiota in mice. Biochemical and Biophysical Research Communications 2018, 495(1): 273-279

\section{ISSUE DATE:}

2018-01-01

URL:

http://hdl.handle.net/2433/228894

\section{RIGHT:}

(c) 2017. This manuscript version is made available under the CC-BY-NC-ND 4.0 license

http://creativecommons.org/licenses/by-nc-nd/4.0/; The full-text file will be made open to the public on 1 January 2019 in accordance with publisher's 'Terms and Conditions for Self-Archiving'; この論文は出版社版でありません。引用の際 には出版社版をご確認ご利用ください。; This is not the published version. Please cite only the published version. 
Supplementation of pancreatic digestive enzymes alters the composition of intestinal microbiota in mice

Hiroki Nishiyama ${ }^{1, *}$, Tomoyuki Nagai ${ }^{2, *}$, Masatoshi $\mathrm{Kudo}^{2}$, Yoshihisa Okazaki ${ }^{2}$, Yoshinao Azuma ${ }^{4}$, Tomohiro Watanabe ${ }^{2}$, Susumu Goto ${ }^{3}$, Hiroyuki Ogata ${ }^{1, \dagger}$, Toshiharu Sakurai $^{2, \dagger}$

${ }^{1}$ Institute for Chemical Research, Kyoto University, Gokasho, Uji, Kyoto, 611-0011, Japan

${ }^{2}$ Department of Gastroenterology and Hepatology, Kindai University Faculty of Medicine, 377-2 Ohno-Higashi, Osaka-Sayama, Osaka, 589-8511, Japan

${ }^{3}$ Database Center for Life Science, Joint Support-Center for Data Science Research, Research Organization of Information and Systems, 178-4-4 Wakashiba, Kashiwa, Chiba, 277-0871, Japan

${ }^{4}$ Biology-Oriented Science and Technology, Kindai University, 930 Nishimitani, Kinokawa, Wakayama 649-6493, Japan

\footnotetext{
* These authors equally contributed to this work.

${ }^{\dagger}$ Co-corresponding authors.
} 


\section{ACCEPTED MANUSCRIPT}

\section{Abstract}

Although pancreatic enzyme replacement therapy (PERT) is effective in the alleviation of pancreatic exocrine insufficiency (PEI)-related symptoms in patients with chronic pancreatitis, its mechanism of action is poorly understood. Recent studies suggest that the intestinal microbiota is associated with the pathogenesis of chronic pancreatitis. Therefore, we hypothesized that PERT exerts its effect by modifying the intestinal microbiota in addition to its presumed role in promoting fat and protein absorption. To explore the mechanism of action of PERT, we analyzed the intestinal microbiotas of two groups of mice treated with either pancrelipase or tap water by using $16 \mathrm{~S}$ rRNA amplicon sequencing. The results revealed that the bacterial compositions of the pancrelipase-treated mice were significantly different from those of the control samples. Akkermansia muciniphila, a key beneficial bacterium in the intestinal tract, showed a higher relative abundance in the pancrelipase-treated samples than in the control samples. Lactobacillus reuteri, a widely used probiotic bacterium known to relieve intestinal inflammation, also showed a higher relative abundance in the pancrelipase-treated samples. These results suggested that PERT induces the colonization of beneficial bacteria, thereby contributing to the attenuation of PEI-associated symptoms in addition to improvement of the nutritional state. 


\section{Keywords}

Chronic pancreatitis; pancreatic exocrine insufficiency; pancreatic enzyme replacement therapy; pancrelipase; intestinal microbiota; Akkermansia muciniphila 


\section{ACCEPTED MANUSCRIPT}

\section{Introduction}

Chronic pancreatitis is a persistent inflammation of the pancreas with pathological findings of the infiltration of immune cells and the development of fibrosis [1]. Clinical courses of patients with chronic pancreatitis are characterized by acute exacerbation and remission phases. Repeated episodes of acute exacerbation cause reduction of the functional pancreatic parenchymal mass through the destruction of the acinar architecture, which leads to the development of fibrosis [1]. Such loss of functional pancreatic parenchymal mass results in impaired functions of both the exocrine and the endocrine pancreas. In fact, patients with advanced stages of chronic pancreatitis exhibit symptoms associated with pancreatic exocrine insufficiency (PEI), such as diarrhea, malabsorption, and steatorrhea [1]. PEI, caused by impaired secretion of pancreatic digestive enzymes due to loss of intact pancreatic acinar cells [1], represents one of the most frequent complications of chronic pancreatitis [2].

Pancreatic enzyme replacement therapy (PERT) is widely used in the treatment of chronic pancreatitis patients exhibiting PEI-associated symptoms and improves fat and protein absorption and serum nutritional parameters in a significant population of these patients [3,4]. Recent clinical trials have shown that PERT results in relief and improvement of PEI-associated symptoms in patients with chronic pancreatitis [5]. 
PERT is generally assumed to alleviate PEI-associated symptoms by restoring pancreatic digestive activity in the gastrointestinal tract. However, the mechanism by which PERT ameliorates PEI-associated symptoms is still poorly defined.

Recent studies have highlighted the involvement of immune responses against intestinal microbiota in the development of chronic pancreatitis [6]. For example, the activation of pattern recognition receptors (i.e., Toll-like receptors and nucleotide-binding oligomerization-like receptors), that detect microbe-associated molecular patterns derived from the intestinal microbiota, have been reported to play a critical role in the development of experimental chronic pancreatitis $[7,8]$. Significant alterations in the intestinal microbiota in patients with chronic pancreatitis have also been reported $[9,10]$. Furthermore, changes in nutritional content within the gastrointestinal tract have been known to be able to alter the homeostatic colonization of commensal microbiota [11]. These studies suggest that nutrient mal-digestion caused by PEI leads to a significant alteration in the intestinal microbiota, which may further worsen chronic pancreatitis through the development of excessive innate immune responses. Therefore, we hypothesized that PERT improves PEI-associated symptoms in chronic pancreatitis not only by restoring pancreatic digestive activity, but also by altering the intestinal microbiota. To test this hypothesis, we used male C57BL/6J mice 
treated with pancrelipase and performed 16S ribosomal RNA gene amplicon analyses of the microbiotas sampled from their cecum, transverse colon, and stool with an aim to understand the effect of PERT on intestinal microbiota. Our findings provide evidence supporting our hypothesis that PERT alleviates PEI-associated symptoms not only by improving digestive activity, but also by altering the composition of the intestinal microbiota in mice.

\section{Materials and Methods}

\subsection{Mice}

We used 8-9-weeks-old male C57BL/6J mice $(25.0 \pm 0.5 \mathrm{~g}$; SLC JAPAN, Inc., Shizuoka, Japan) and divided them into 2 groups: control and pancrelipase-treated groups. The control group was given tap water at the dose of $0.75 \mathrm{~mL} /$ day divided into 3 parts for 21 days. The pancrelipase-treated group was treated with pancrelipase-a commercial mixture of pancreas amylase, lipase, and protease (chymotrypsin) (Lipacreon, EA pharma, Japan) — at a dose of $1.2 \mathrm{mg} /$ day divided into 3 parts for 21 days. After 21 days, the mice were killed, and their cecum, transverse colon, and stool were collected, frozen, and stored at $-20^{\circ} \mathrm{C}$. All animal experiments were conducted 
according to the ethical guidelines of the review boards of Kindai University Faculty of

Medicine, and the animal experiments were approved by the same review boards.

\subsection{DNA extraction and sequencing}

The frozen cecum, transverse colon, and stool samples were thawed and homogenized using Zirconia/Silica Beads (BioSpec Products) in a MagNALyzer (Roche Diagnostics). Following homogenization, DNA was extracted using the QIAamp DNA Stool Mini Kit and the QIAamp DNA Mini Kit according to the manufacturer's instructions (Qiagen GmbH, Hilden Germany). The extracted DNA samples were used as the template in PCR for amplification of the variable V3-V4 16S rRNA gene regions with $16 \mathrm{~S}$ Amplicon PCR Forward primer 5'-TCGTCGGCAGCGTCAGATGTGTATAAGAGACAG-MID-GT-CCTACGGGNG GCWGCAG-3' and 16S Amplicon PCR Reverse primer 5'-GTCTCGTGGGCTCGGAGATGTGTATAAGAGACAG-MID-GT-GACTACHVG GGTATCTAATCC-3'.

Sequencing libraries were prepared using the " $16 \mathrm{~S}$ Metagenomic Sequencing Library Preparation: Preparing 16S Ribosomal RNA Gene Amplicons for the Illumina MiSeq System' protocol [12] with the use of the Nextera XT Index Kit (Illumina). Sequencings were performed using the MiSeq Reagent Kit v2 (300 cycles) and MiSeq 
(Illumina, San Diego, CA, USA) device according to the manufacturer's recommendations.

\subsection{Preprocessing of sequence data}

Low-quality sequence regions were trimmed from each paired-end read using Trimmomatic (version 0.35) (SLIDINGWINDOW:40:15, MINLEN:50) [13]. Primer sequences were trimmed from paired-end reads using Cutadapt (version 1.11) (-e 0.06, -pair-filter=both) [14]. The resulting trimmed paired-end reads were merged using FLASh (version 1.2.1) (-m 30, -M 271, -x 0.25) [15]. These merged reads are referred to as the "reads" in the rest of the manuscript. Raw sequence data were submitted to DDBJ/DRA under the accession number DRA006124. The reads are available from ftp://ftp.genome.jp/pub/db/community/microbiome_kindai.

\subsection{Generation of operational taxonomy units}

The following analyses were conducted using programs included in the Quantitative Insights Into Microbial Ecology (QIIME version 1.9.1) software [16], unless stated otherwise. The analyses used the representatives of 16S rRNA sequences from the Greengenes database (version 13_5) pre-clustered at 97\% sequence identity threshold [17]. These representative sequences are referred to as the "reference sequences" in the rest of the manuscript. 


\section{ACCEPTED MANUSCRIPT}

The reads were each clustered against the reference sequences at $97 \%$ sequence identity threshold to form operational taxonomic units (OTUs) using parallel_pick_otus_uclust_ref.py. Reads that did not match with the reference sequences at the $97 \%$ sequence identity threshold were excluded from the analysis. A taxonomic classification was assigned to each OTU by referring to the taxonomy of the reference sequence included in the OTU. The resulting OTU table represents OTU identifiers, assigned taxonomic classifications, and read counts for each sample.

\subsection{Assessment of alpha diversity}

Alpha diversity, the diversity within a community, was assessed by rarefaction curves as well as by Shannon's diversity index, the latter of which considers both the richness and evenness of the community structure [18]. Rarefaction curves were generated by averaging the OTU counts from 10 times of random re-sampling of reads at different depths with intervals of 5,000 sequences using parallel_multiple_rarefactions.py. For calculating Shannon's diversity index, each set of reads was rarefied down to 57,762 reads (i.e., the number of reads for the smallest sample) using single_rarefaction.py. Statistical significances of the differences in Shannon's index values between the samples were tested using Welch's t-test (with a significance level of 0.05 without correction for multiple test) implemented in SciPy 
[19].

2.6. Assessment of beta diversity

Beta diversity, the dissimilarity between communities, was measured using the weighted UniFrac distance [20], which takes into account the relative abundances of each OTU and their phylogenetic relationships. The reference sequences corresponding to each OTU were first aligned against a template alignment provided by QIIME using PyNAST [21] implemented in align_seqs.py. The resulting alignment was then used to create a phylogenetic tree using FastTree [22] implemented in make_phylogeny.py. Each set of reads was rarefied down to 57,762 reads using single_rarefaction.py. The weighted UniFrac distances between samples were calculated using parallel_beta_diversity.py, together with the phylogenetic tree and the rarefied set of reads.

\subsection{Identification of over-/under-represented bacterial taxa}

To identify bacterial taxa that are differentially abundant (i.e., either over- or under-represented) in a set of samples relative to another set of samples, we first grouped OTUs at the species level according to their assigned taxa and generated a taxonomy table, which records the read counts for each taxon across samples, using summarize_taxa.py. OTUs with taxonomic assignments at only genus or higher 


\section{ACCEPTED MANUSCRIPT}

taxonomic levels were grouped at the lowest level of taxonomy that they received.

Bacterial taxa with the assignment of less than 10 reads were omitted from analysis using filter_otus_from_otu_table.py. Statistical significance of the difference in the relative abundance of a taxon between groups of samples (i.e., differential abundance between control vs. pancrelipase-treated samples) was assessed using the DESeq2 negative binomial Wald test [23] implemented in differential_abundance.py. False discovery rate (q-value) was controlled at 0.1 based on the obtained p-values with the use of the Benjamini-Hochberg correction as implemented in DESeq2. For each bacterial taxon showing a significant difference in its relative abundance between two sample groups (i.e., q-value $<0.1$ ), we further examined the sample group showing the higher average relative abundance. If one or more of the samples in this group contained no reads, we did not consider the taxon over- or under-represented.

\section{Results}

\subsection{Generation of OTUs}

After quality control, we obtained a total of 5,167,710 merged reads (Table S1). Of these, 3,572,866 reads $(69.13 \%)$ matched with reference sequences in the Greengenes database and formed 2,692 OTUs (corresponding to 31 orders, 54 families, 62 genera, and 19 species of bacteria). These results were organized into an OTU table used for the 
following analyses.

\subsection{Pancrelipase treatment did not induce significant alpha diversity changes}

There were no systematic differences in the bacterial community richness (i.e., the number of OTUs in a sequencing depth normalized sample) between the control and the pancrelipase-treated group in any of the cecum, transverse colon, and stool samples (Figure S1A). Furthermore, there were no statistically significant differences in Shannon's diversity index between the control and pancrelipase-treated samples (Welch's t-test, Figure S1B). Pancrelipase treatment thus had no considerable influence on the alpha diversity of the tested intestinal microbiotas.

\subsection{Pancrelipase treatment induced alterations in bacterial community composition}

The bacterial composition of the pancrelipase-treated samples at the phylum level were clearly different from those of the control samples (Figure 1). A major difference was the increased relative abundances of Verrucomicrobia in the pancrelipase-treated samples. This phylum showed extensive increase in the pancrelipase-treated samples (control vs. pancrelipase, $0.13 \%$ vs. $7.58 \%$ on average). All the OTUs assigned to Verrucomicrobia corresponded to Akkermansia muciniphila. Another subtle difference was the lower Firmicutes to Bacteroidetes ratio in the transverse colon samples compared with that in the controls, although the difference was not statistically 
significant (Welch's t-test, $\mathrm{p}=0.0623$ ). Principal coordinate analysis was further used to visualize compositional differences at a finer scale (i.e., at the OTU level) based on the weighted UniFrac distance (Figure 2). The resulting three-dimensional plot showed that the spatial distribution of the pancrelipase-treated samples was distinct from that of the control samples.

\subsection{Bacterial taxa showing significant changes in their relative abundances}

By re-grouping the 2,692 OTUs according to their taxonomic classifications, we obtained a taxonomy table recording read counts for 106 bacterial taxa for each samples. Of these, 51 bacterial taxa represented by less than 10 reads were excluded from the analysis. Comparison between the control and pancrelipase-treated samples revealed 17 instances that had satisfied our criteria (see Materials and Methods) (Figure 3). In eight of these instances, the bacterial taxa were over-represented (i.e., higher relative abundance) in the pancrelipase-treated samples. These corresponded to Akkermansia muciniphila (cecum, transverse colon, and stool), Alcaligenaceae Sutterella (transverse colon), Lactobacillus reuteri (cecum), Clostridiaceae Clostridium (transverse colon), and Erysipelotrichaceae Coprobacillus (cecum and stool). In the remaining nine instances, the bacterial taxa were under-represented (i.e., lower relative abundance) in the pancrelipase-treated samples than in the control samples. These taxa were 
Desulfovibrionales Desulfovibrionaceae (transverse colon), Desulfovibrionaceae

Desulfovibrio (transverse colon), Desulfovibrionaceae Bilophila (transverse colon, stool), Clostridales Lachnospiraceae (transverse colon and stool), Lachnospiraceae Dorea (transverse colon and stool) and Clostridiales Mogibacteriaceae (transverse colon). Collectively, these results suggested that pancrelipase treatment induced a statistically significant compositional shift in the mouse intestinal microbiota.

\section{Discussion}

PERT has been established as an effective treatment for PEI in chronic pancreatitis.

However, the mechanism by which PERT ameliorates PEI-associated symptoms is still poorly defined. We hypothesized that PERT exerts its effect by modifying the intestinal microbiota, and showed that there were significant differences in the compositions of mouse intestinal microbiotas between pancrelipase-treated and control samples.

Of the significant differences in the relative abundances of bacterial taxa between the pancrelipase-treated and control samples, the difference in the relative abundance of Akkermansia muciniphila (belonging to Verrucomicrobia) was the most striking. Indeed, the average relative abundance of $A$. muciniphila was 58-fold higher in the pancrelipase-treated samples than in the control samples. One of the biggest risk factors 
for chronic pancreatitis is alcohol drinking, which impairs intestinal barrier function, followed by translocation of intestinal bacteria [24]. A. muciniphila is known to degrade intestinal mucin into propionic and acetic acids, which promote beneficial microbe interactions in the intestinal tract [25]. In line with this, Garnder et al. previously reported that ethanol-induced intestinal barrier dysfunction is associated with a prominent decline of A. muciniphila [26]. Furthermore, supplementation of A. muciniphila was reported to enhance intestinal barrier function by promoting mucus thickness and tight junction protein expression [26]. Therefore, it is possible that pancrelipase attenuates PEI-associated symptoms by inducing colonization of A. muciniphila, followed by normalization of intestinal barrier function. However, it should be noted that whether pancrelipase treatment induces the colonization of this bacterium in chronic pancreatitis patients and beneficial effects remains to be determined.

Four other bacteria were also over-represented in the pancrelipase-treated samples. One of these bacteria was Lactobacillus reuteri, a well-known probiotic bacterium. In fact, $L$. reuteri has been proven to relieve intestinal inflammation by converting L-histidine to histamine, which suppresses the host immune system by activating the $\mathrm{H} 2$ receptor [27]. The ability of $L$. reuteri to prevent or alleviate colitis in mice has also been reported $[28,29]$. Thus, it is possible that pancrelipase-induced colonization of $L$. 
reuteri contributes to the maintenance of intestinal immune homeostasis. Alcaligenaceae Sutterella, Clostridiaceae Clostridium, and Erysipelotrichaceae Coprobacillus also showed elevated relative abundances in the pancrelipase-treated samples, but the effect of colonization of these bacteria on host intestinal functions has not been clarified [30,31]. With regard to Clostridiaceae Clostridium, the genus contains both pro-inflammatory bacteria (Clostridium difficile [32]) and anti-inflammatory bacteria (e.g., Clostridium butyricum MIYARI 588 [33]). Together, these results indicated that $A$. muciniphila, with its ability to promote intestinal barrier function, and L. reuteri, with its ability to regulate inflammation, showed increased relative abundances in the colon and stool samples of the pancrelipase-treated mice. The colonization of such beneficial bacteria induced by pancrelipase treatment may partially explain the mechanisms by which PERT attenuates PEI-associated symptoms.

We also identified six bacterial taxa under-represented in the pancrelipase-treated mice. Species belonging to the Bilophila and Desulfovibrio genera of the family Desulfovibrionaceae might have colitogenic functions, since they produce hydrogen sulfide, which promotes intestinal inflammation in rats when administered at amounts exceeding the capacity of colonocytes to detoxify it $[34,35,36]$. Lachnospiraceae Dorea also showed lower relative abundance in the pancrelipase-treated samples. Certain 


\section{ACCEPTED MANUSCRIPT}

members of the Lachnospiraceae family are known to produce butyric acid, which promotes intestinal epithelial barrier function [37]. Clostridiales Mogibacteriaceae also showed decreased relative abundances in the pancrelipase-treated mice, but the effect of colonization of these bacteria on host intestinal functions has not been clarified.

A few studies have previously investigated the composition of intestinal microbiotas in patients with chronic pancreatitis or in model mice $[9,38,39]$. Jandhyala et al. observed significant decreases in the abundances of Faecalibacterium prausnitzii and Ruminococcus bromii and an increase in the Firmicutes to Bacteroidetes ratio in the intestinal microbiomes of chronic pancreatitis patients compared with that in healthy subjects. However, in our study, we could not find any significant pancrelipase-induced changes in these species or in the Firmicutes to Bacteroidetes ratio between the two groups of mice. Helicobacter pylori is a suspected pathogen of autoimmune pancreatitis (i.e., a rare form of chronic pancreatitis) [40]. In our study, the relative abundance of $H$. pylori was found to be very low $(<0.0035 \%)$ in the intestinal microbiotas of the mice and showed no significant changes upon pancrelipase treatment.

Furthermore, several previous studies using chronic pancreatitis model mice have reported increases and decreases in the relative abundance of several bacterial taxa in the model mice relative to that in the control mice [38,39]. In contrast, most of these 


\section{ACCEPTED MANUSCRIPT}

taxa did not show any significant changes in their relative abundance upon pancrelipase treatment in our study, except for Lactobacillus and Lachnospiraceae. These bacteria have been previously reported to show decreased relative abundances in chronic pancreatitis model mice; however, the relative abundance of Lactobacillus reuteri was over-represented in the cecum samples of the pancrelipase-treated mice and that of Lachnospiraceae was under-represented in the transverse colon and stool samples of the pancrelipase-treated mice. Overall, the administration of pancrelipase did not induce microbial changes akin to those observed in chronic pancreatitis patients or its model mice, with the sole exception of the under-representation of Lachnospiraceae.

Future studies on intestinal microbiotas derived from chronic pancreatitis model mice and patients with or without PEI need to be conducted to better understand the mechanism by which pancrelipase ameliorates the symptoms of chronic pancreatitis.

To the best of our knowledge, our study is the first to reveal that oral supplementation of pancrelipase significantly alters the intestinal microbiota in mice. Our findings supports the idea that pancrelipase exerts its effect in PERT by modifying the gut microbiota, in addition to its presumed effect of improving the nutritional state of patients. Furthermore, the fact that the relative abundances of A. muciniphila and $L$. reuteri-two microorganisms that are known to be beneficial to the maintenance of the 
intestinal barrier-increased upon pancrelipase administration in the intestinal microbiota of the mice suggests that new possibilities for the treatment of chronic pancreatitis, such as probiotics and fecal transplantation, should be considered. 


\section{Acknowledgements}

This work was in part supported by JSPS/KAKENHI (17K09396), JSPS/KAKENHI (No. 16KT0020), the Naito Foundation, the SENSHIN Medical Foundation, the Yakult

Bioscience Foundation, the Smoking Research Foundation, and by Japan Agency for Medical Research and Development (AMED) Grants for Research on Intractable Diseases. Computational work was completed at the Super Computer System, Institute for Chemical Research, Kyoto University.

\section{Conflict of interest}

Pancrelipase was provided by EA Pharma Co., Ltd. 


\section{ACCEPTED MANUSCRIPT}

\section{References}

[1] J. M. Braganza, S. H. Lee, R. F. McCoy, et al., Chronic pancreatitis, Lancet 377 (2011) 1184-1197.

[2] T. C. Hall, G. Garcea, M. A. Webb, et al., The socio-economic impact of chronic pancreatitis: a systematic review, J. Eval. Clin. Pract. 20 (2014) 203-207.

[3] D. C. Whitcomb, A. Bodhani, K. Beckmann, et al., Efficacy and Safety of Pancrelipase/Pancreatin in Patients With Exocrine Pancreatic Insufficiency and a Medical History of Diabetes Mellitus, Pancreas 45 (2016) 679-686.

[4] J. G. D’Haese, G. O. Ceyhan, I. E. Demir, et al., Pancreatic Enzyme Replacement Therapy in Patients With Exocrine Pancreatic Insufficiency Due to Chronic Pancreatitis: A 1-Year Disease Management Study on Symptom Control and Quality of Life, Pancreas 43 (2014) 834-841.

[5] D. de la Iglesia-García, W. Huang, P. Szatmary, Efficacy of pancreatic enzyme replacement therapy in chronic pancreatitis: systematic review and meta-analysis, Gut 66 (2017) 1354-1355.

[6] T. Watanabe, M. Kudo, W. Strober, Immunopathogenesis of pancreatitis, Mucosal Immunol. 10 (2017) 283-298.

[7] A. Ochi, A. H. Nguyen, A. S. Bedrosian, et al., MyD88 inhibition amplifies dendritic 


\section{ACCEPTED MANUSCRIPT}

cell capacity to promote pancreatic carcinogenesis via Th2 cells, J. Exp. Med. 209 (2012) 1671-1687.

[8] T. Watanabe, Y. Sadakane, N. Yagama, et al., Nucleotide-binding oligomerization domain 1 acts in concert with the cholecystokinin receptor agonist, cerulein, to induce IL-33-dependent chronic pancreatitis, Mucosal Immunol. 9 (2016) 1234-1249.

[9] S. M. Jandhyala, A. Madhulika, G. Deepika, et al., Altered intestinal microbiota in patients with chronic pancreatitis: implications in diabetes and metabolic abnormalities, Sci. Rep. 7 (2017) 43640.

[10] M. Signoretti, R. Roggiolani, C. Stornello, et al., Gut microbiota and pancreatic diseases, Minerva Gastroenterol. Dietol. 63 (2017) 399-410.

[11] M. Levy, A. A. Kolodziejczyk, C. A. Thaiss, Dysbiosis and the immune system, Nat. Rev. Immunol. 17 (2017) 219-232.

[12] Illumina, 16S Metagenomic Sequencing Library Preparation, http://jp.support.illumina.com/downloads/16s_metagenomic_sequencing_library_prepar ation.html, 2013 (accessed 15/July/2015).

[13] A. M. Bolger, M. Lohse, B. Usadel, Trimmomatic: a flexible trimmer for Illumina sequence data, Bioinformatics 30 (2014) 2114-2120.

[14] M. Martin, Cutadapt removes adapter sequences from high-throughput sequencing 


\section{ACCEPTED MANUSCRIPT}

reads, EMBnet.journal 17 (2011) 10-12.

[15] T. Magoč, S. L. Salzberg, FLASH: fast length adjustment of short reads to improve genome assemblies, Bioinformatics 27 (2011) 2957-2963.

[16] J. G. Caporaso, J. Kuczynski, J. Stombaugh, et al., QIIME allows analysis of high-throughput community sequencing data, Nat. Methods 7 (2010) 355-356.

[17] D. McDonald, M. N. Price, J. Goodrich, et al., An improved Greengenes taxonomy with explicit ranks for ecological and evolutionary analyses of bacteria and archaea, ISME J. 6 (2012) 610-618.

[18] C. E. Shannon, A Mathematical Theory of Communication, Bell Syst. Tech. J. 27 (1948) 379-423.

[19] E. Jones, E Oliphant, P. Peterson, et al., SciPy: Open Source Scientific Tools for Python, 0.16.1, 2001-. http://www.scipy.org/.

[20] C. Lozupone, R. Knight, UniFrac: a New Phylogenetic Method for Comparing Microbial Communities, Appl. Environ. Microbiol. 71 (2005) 8228-8235.

[21] J. G. Caporaso, K. Bittinger, F. D. Bushman, et al., PyNAST: a flexible tool for aligning sequences to a template alignment, Bioinformatics 26 (2010) 266-267.

[22] M. N. Price, P. S. Dehal, A. P. Arkin, FastTree 2 - Approximately Maximum-Likelihood Trees for Large Alignments, PLoS One 5 (2010) e9490. 


\section{ACCEPTED MANUSCRIPT}

[23] M. I. Love, W. Huber, S. Anders, Moderated estimation of fold change and dispersion for RNA-seq data with DESeq2, Genome Biol. 15 (2014) 550.

[24] A. Vonlaufen, L. Spahr, M. V. Apte, et al., Alcoholic pancreatitis: A tale of spirits and bacteria, World J. Gastrointest. Pathophysiol. 5 (2014) 82-90.

[25] W. M. de Vos, Microbe Profile: Akkermansia muciniphila: a conserved intestinal symbiont that acts as the gatekeeper of our mucosa, Microbiology 163 (2017) 646-648.

[26] C. Grander, T. E. Adolph, V. Wieser, et al., Recovery of ethanol-induced Akkermansia muciniphila depletion ameliorates alcoholic liver disease, Gut (2017) doi: 10.1136/gutjnl-2016-313432.

[27] C. Gao, A. Major, D. Rendon, et al., Histamine H2 Receptor-Mediated Suppression of Intestinal Inflammation by Probiotic Lactobacillus reuteri, mBio 6 (2015) e01358-15.

[28] A. R. Mackos, T. D. Eubank, N. M. A. Parry, et al., Probiotic Lactobacillus reuteri Attenuates the Stressor-Enhanced Severity of Citrobacter rodentium Infection, Infect. Immun. 81 (2013) 3253-3263.

[29] A. R. Mackos, J. D. Galley, T. D. Eubank, et al., Social stress-enhanced severity of Citrobacter rodentium-induced colitis is CCL2-dependent and attenuated by probiotic Lactobacillus reuteri, Mucosal Immunol. 9 (2016) 515-526. 


\section{ACCEPTED MANUSCRIPT}

[30] A. Labbé, J. G. Ganopolsky, C. J. Martoni, et al., Bacterial Bile Metabolising Gene

Abundance in Crohn's, Ulcerative Colitis and Type 2 Diabetes Metagenomes, PLoS One, 9 (2014) e115175.

[31] P. Palvidis, N. Powell, R. P. Vincent, et al., Systematic review; bile acids and intestinal inflammation-luminal aggressors or regulators of mucosal defence, Aliment. Pharmacol. Ther. 42 (2015) 802-817.

[32] M. C. Abt, P. T. McKenney, E. G. Pamer, Clostridium difficile colitis: pathogenesis and host defence, Nat. Rev. Microbiol. 14 (2016) 609-620.

[33] A. Hayashi, T. Sato, N. Kamada, et al., A Single Strain of Clostridium butyricum Induces Intestinal IL-10-Producing Macrophages to Suppress Acute Experimental Colitis in Mice, Cell Host Microbe 13 (2013) 711-722.

[34] Y. A. Warren, D. M. Citron, C. Y. Merriam, et al., Biochemical differentiation and comparison of Desulfovibrio species and other phenotypically similar genera, J. Clin, Microbiol. 43 (2005) 4041-4045.

[35] S. B. Singh, H. C. Lin, Hydrogen Sulfide in Physiology and Diseases of the Digestive Tract, Microorganisms 3 (2015) 866-889.

[36] M. Beaumont, M. Andriamihaja, A. Lan, et al., Detrimental effects for colonocytes of an increased exposure to luminal hydrogen sulfide: The adaptive response, Free 
Radic. Biol. Med. 93 (2016) 155-164.

[37] C. J. Meehan, R. G. Beiko, A phylogenomic view of ecological specialization in the Lachnospiraceae, a family of digestive tract-associated bacteria, Genome Biol. Evol. 6 (2014) 703-713.

[38] Y. Hu, C. Teng, S. Yu, et al., Inonotus obliquus polysaccharide regulates gut microbiota of chronic pancreatitis in mice, A.M.B. Express 7 (2017) doi: 10.1186/s13568-017-0341-1.

[39] K. Li, C. Zhuo, C. Teng, et al., Effects of Ganoderma lucidum polysaccharides on chronic pancreatitis and intestinal microbiota in mice, Int. J. Biol. Macromol. 93 (2016) 904-912.

[40] F. Guarneri, C. Guarneri, and S. Benvenga, Helicobacter pylori and autoimmune pancreatitis: role of carbonic anhydrase via molecular mimicry?, J. Cell Mol. Med. 9 (2005) 741-744. 


\section{Figure legends}

Figure 1. Relative abundances of different bacterial taxa at the phylum level.

Figure 2. Comparison of bacterial communities across samples. (A) OTU-level comparisons were performed for all control (blue) and pancrelipase-treated (red) samples by using principal coordinate analysis based on the weighted UniFrac distance. Results for the cecum (B), transverse colon (C), and stool (D) samples were plotted separately for better visualization.

Figure 3. Bacterial taxa showing significantly different relative abundances between the control and pancrelipase-treated samples collected from the cecum $(\mathrm{C})$, transverse colon (T), or stool (S). Relative abundances in the control and pancrelipase-treated samples are represented by blue and red bars, respectively.

\section{Supplementary data}

Table S1. Number of reads in samples at different steps of analyses.

Figure S1. Comparison of alpha diversity across samples. (A) Rarefaction curves for the number of OTUs for samples from the control and pancrelipase-treated mice. (B) Shannon's diversity index values for samples from the control (Ctrl, blue) and pancrelipase-treated (Panc, red) mice. 
Relative abundance of bacterial taxa (\%)
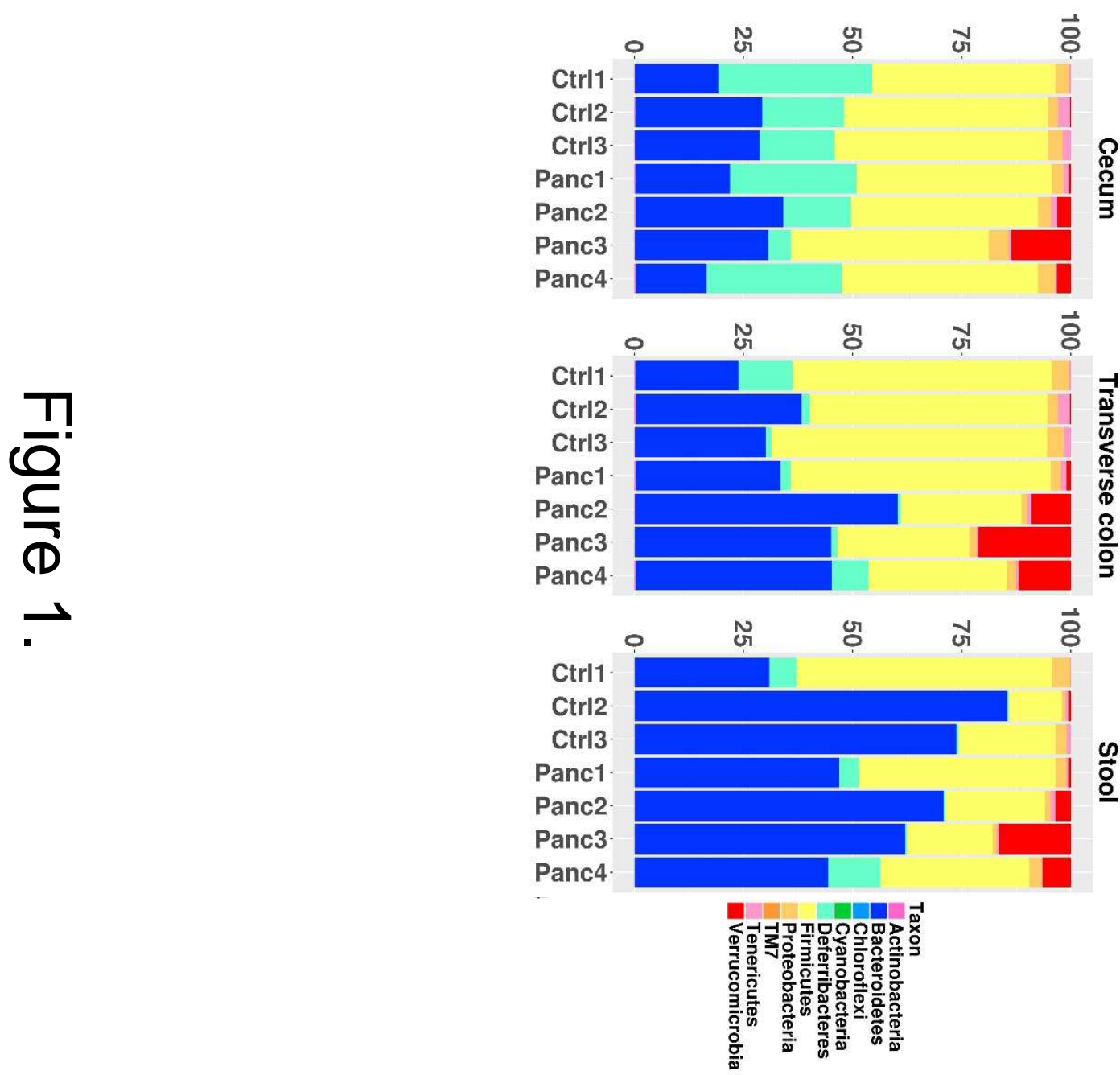

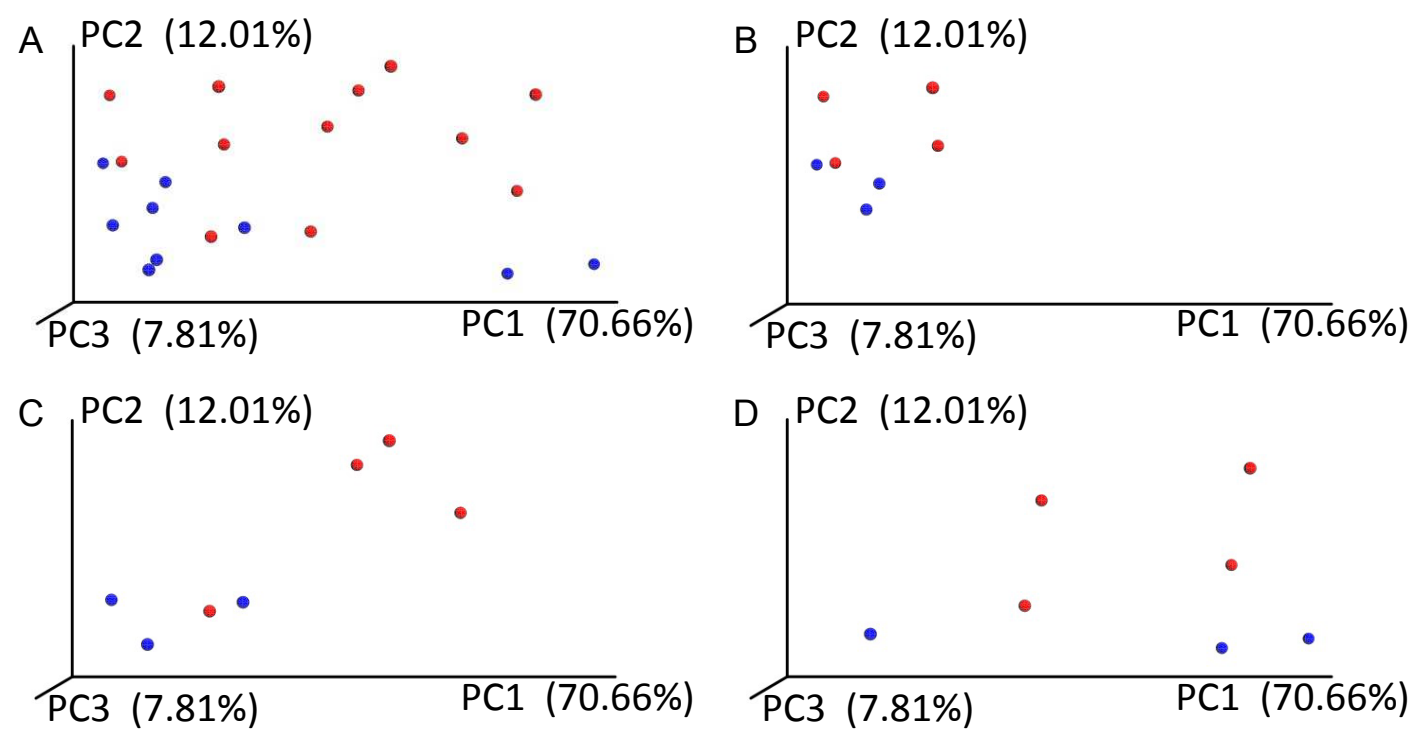

Figure 2. 
Relative abundance of bacterial taxa (\%)
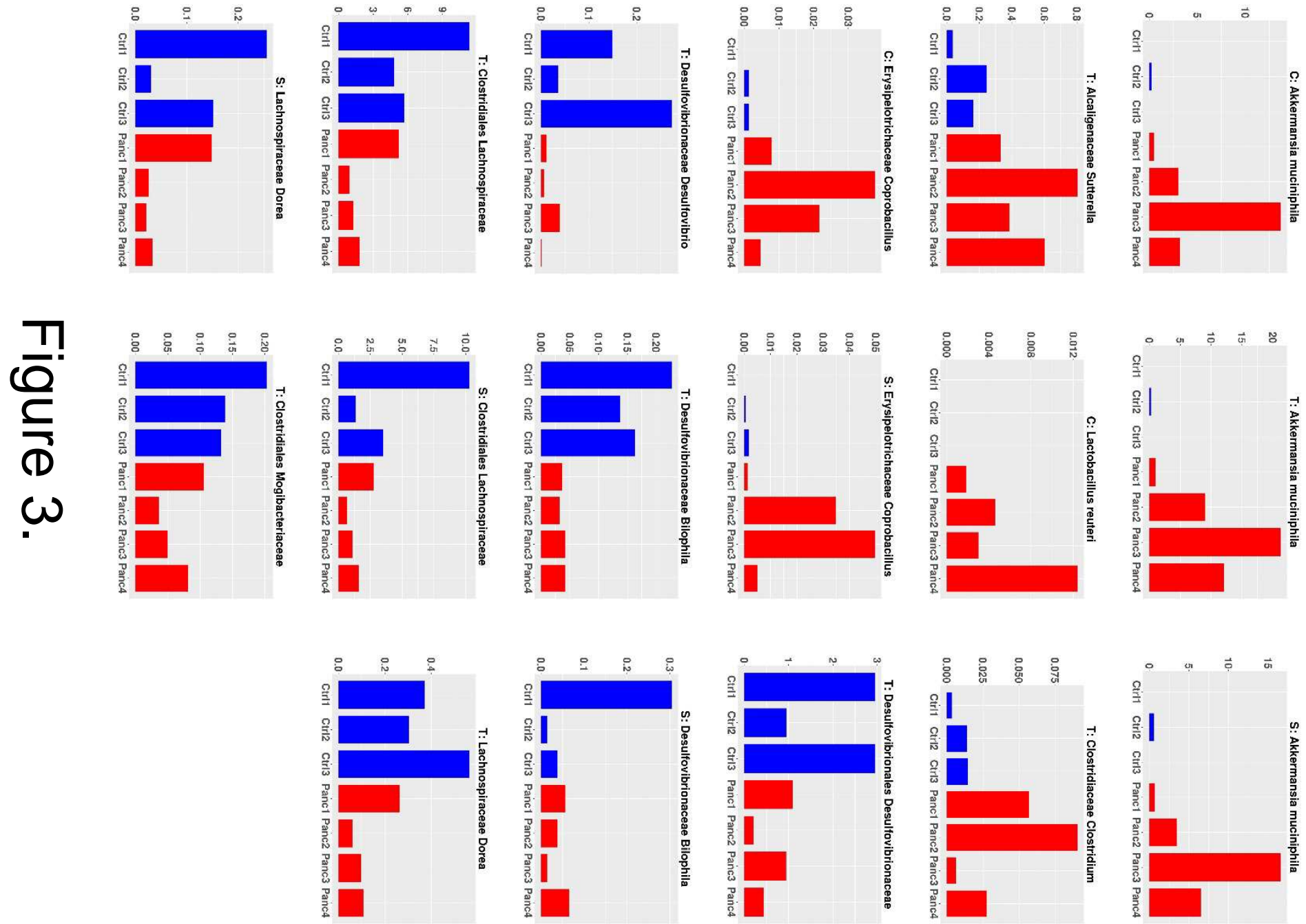


\section{Highlight}

- Oral supplementation of pancrelipase alters composition of intestinal microbiota.

- Over-represented taxa include bacteria promoting intestinal homeostasis.

- Akkermansia muciniphila and Lactobacillus reuteri are over-represented.

- Pro-inflammatory bacteria is inhibited by supplementation of pancrelipase.

- Pancrelipase may help treat chronic pancreatitis by altering intestinal microbiota. 